\title{
Fuzzy logic as support for security and safety solution in soft targets
}

\author{
Lucia Ďuricová ${ }^{1}$ and Martin Hromada ${ }^{2}$ \\ Tomas Bata University in Zlin, Faculty of applied informatics, Nad Stranemi 4511, Zlin, 76005, Czech Republic
}

\begin{abstract}
Security and safety situations in objects, which are categorized as soft targets, is difficult. The current solving is based on several different type of solving. Soft targets are specific objects, and it requires special software solution. The proposal is based on fuzzy logic. Fuzzy logic could apply more expert's knowledges and it could help owners and managers with adequate responses in critical situation, and also definition of adequate preventive actions. System solving could help effectivity of proposed measures. The decision making is based on this fuzzy logic support and aim is explained in paper.
\end{abstract}

\section{Fuzzy logic}

Fuzzy logic is based on fuzzy set theory which is generalization of the classical set theory [8]. The classical sets are also called clear sets, as opposed to fuzzy, and by the same token classical logic id also known as Boolean logic or binary logic. In fuzzy logic are used to examining whole range of values. In classical theory are identified only two states. The first is when element belongs to group, or the second element doesn't belong to group.

\section{Support for decision making}

In security and safety sectors special links are identified which could have impact to developing security situation in object. This special links and relations have special conditions which could be some different in different kinds of objects. Soft targets are specified objects which don't have to implemented special security solution in process. This objects have specific properties which could make reaction more difficult. This properties are identified in next table 1.

Table 1. The properties of soft targets object.

\begin{tabular}{|c|c|}
\hline The property & The specification \\
\hline Public & $\begin{array}{c}\text { Object has in same time } \\
\text { and at the same bordered } \\
\text { area or place a lot of } \\
\text { uncontrolled moving } \\
\text { visitors. }\end{array}$ \\
\hline Distinction & $\begin{array}{c}\text { Objects has different aim } \\
\text { and it relate with interest } \\
\text { groups of visitors. }\end{array}$ \\
\hline Integration & $\begin{array}{c}\text { Object don't have to } \\
\text { integrate special technics }\end{array}$ \\
\hline a
\end{tabular}

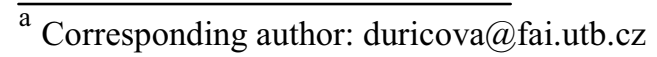

\begin{tabular}{|c|c|}
\hline \multirow{7}{*}{ Localization } & $\begin{array}{c}\text { which could help experts } \\
\text { with solving security } \\
\text { crises. Experts don't have } \\
\text { to know situation in } \\
\text { object. The training of } \\
\text { intervention is vulnerable. }\end{array}$ \\
\hline $\begin{array}{c}\text { Objects cannot be linking } \\
\text { with other object in group } \\
\text { and efficiency of } \\
\text { measures is nod adequate. }\end{array}$ \\
\hline
\end{tabular}

In fuzzy logic are known fuzzy reasoning which is known as approximate reasoning. Reasoning is based on fuzzy rules that are expressed in natural language using linguistic variables which definition have been given above. A fuzzy rule has the form:

If $\mathrm{x} \in \mathrm{A}$ and $\mathrm{y} \in \mathrm{B}$ then $\mathrm{z} \in \mathrm{C}$, with $\mathrm{A}, \mathrm{B}$, and $\mathrm{C}$ fuzzy sets.

For example for one specified object:

If (safety measures are high) and (security option are medium) then (solving in object is secure).

The variable "solving" belongs to the fuzzy set "secure". The degree depends on the degree of validity of the premise, i.e. the membership degree of the variable "safety" to the fuzzy set "high" and membership degree of the variable "security" to fuzzy set "medium". The underlying idea is that more prepositions in premise are checked, the more the suggest output actions must be applied. To determine the degree of truth of the proposition fuzzy "solving will be secure", the fuzzy implication must be defined.

The fuzzy system designer must choose among the wide choice of fuzzy implications already defined, or set it by hand. In the security and safety proposal fuzzy implications are proposed which will be setting by hand.

The result of the application of a fuzzy rule depends on three factors: 
1. The definition of fuzzy implication which were chosen.

2. The definition of membership function, of the fuzzy set, of the proposition located, at the conclusion of the fuzzy rule.

3. The degree of validity of propositions located premise.

When fuzzy operators (and, or, not) are defined, the premise of fuzzy rule may be formed from a combination of fuzzy propositions. All the rules of a fuzzy system is called the decision matrix. On the table 2 are defined examples for security and safety proposal.

Table 2. Examples for decision matrix.

\begin{tabular}{|c|c|}
\hline Fuzzy statement & Result \\
\hline $\begin{array}{c}\text { If (safety requirements are } \\
\text { high) and (safety measures are } \\
\text { low) }\end{array}$ & $\begin{array}{c}\text { Then (situation in } \\
\text { object is } \\
\text { hazardous). }\end{array}$ \\
\hline $\begin{array}{c}\text { If (safety requirements are } \\
\text { medium) and (safety measures } \\
\text { are medium) }\end{array}$ & $\begin{array}{c}\text { Then (situation in } \\
\text { object is } \\
\text { optimising). }\end{array}$ \\
\hline If (safety requirements are & $\begin{array}{c}\text { Then (implemented } \\
\text { measures must be } \\
\text { medium) and (security }\end{array}$ \\
requirements are medium) & $\begin{array}{c}\text { men (reaction must } \\
\text { be implementation } \\
\text { if ( implemented measures must } \\
\text { be high) and (risk of incident is } \\
\text { high) }\end{array}$ \\
\hline \multicolumn{2}{|c|}{ measures). } \\
\hline
\end{tabular}

Fuzzy statement defines specific area of values and operators between this statements. Result consider of evaluation fuzzy statements and definition fuzzy implications. The result can help experts with making decision in crises and difficult situations.

\subsection{The defuzzification in the proposal}

For defuzzification is possible chosen among several possible definitions of defuzzification. In this paper will be presented some primary methods of defuzzification.

Defuzzification is based on converting fuzzy grade to crisp output. It is last part of this process.

The first method is often called Center of area or Center of gravity. We can see it on Figure 1.

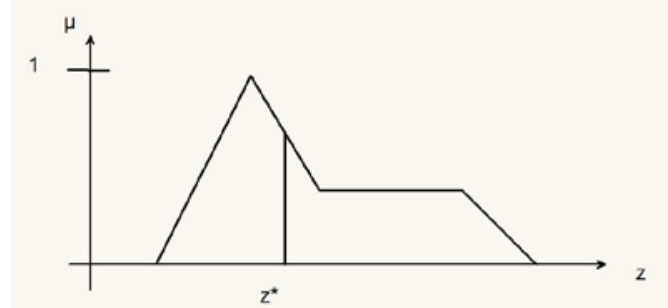

Figure 1. Defuzzification with centre of gravity.

This method for defuzzification is possible formulates with next equation.

$$
z^{*}=\frac{\int \mu_{c}(z) \times z d z}{\int \mu_{c}(z) d z}
$$

The next method is known as height method and based on max-membership principal which we can see on Figure 2.

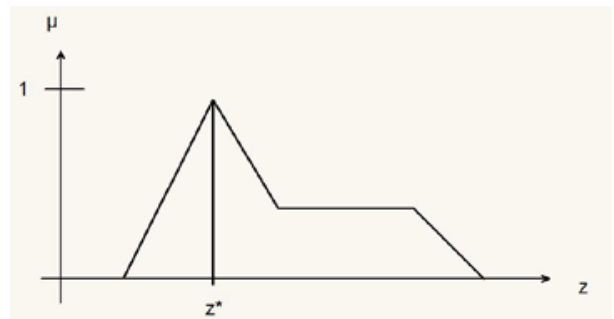

Figure 2. Defuzzification with heigh method.

The heigh method is possible formulates with next equation.

$$
\mu_{c}\left(z^{*}\right) \geq \mu_{c}(z) \text { for all } z \in Z
$$

The next defuzzification method is based on weighted average method which is valided for symmetrical output membership functions. On the figure 3 we can see graph, which formed maximum membership value in each functions.

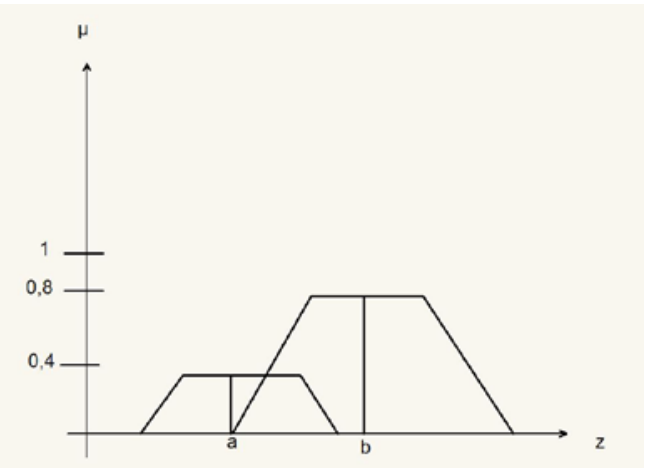

Figure 3. Defuzzification with weighted avarage method.

The next equipment formulates principle of this defuzzification method.

$$
z^{*}=\frac{\sum \mu_{c}(\bar{z}) \times \bar{z}}{\sum \mu_{c}(\bar{z})}
$$

In the next equipment is written model of example which is on grpah.

$$
z^{*}=\frac{a(0,4)+b(0,8)}{0,4+0,8}
$$

In this process is possible used to more methods, which aren't described in paper. For example:

- Middle of maxima.

- Center of sums.

- Center of largest area.

- $\quad$ First or last of maxima.

\section{The proposal of software solving}

The software solving is supported program for decision making in objects in category soft targets. Soft targets are objects which has specifics with common characteristic point:

- A lot of people in the same time at the same place. 
- Object without specific safety and security requirements for object (specific requirements are defined in fire protection category, and it is requiring from law requirements).

- $\quad$ The people moving isn't manage and monitoring depends upon manager or owner of object.

- The intervention of integrated emergency system is not regularly rehearsed.

The aim of this system is supporting experts and visualization possible scenarios in next incidents. This system may define level of measures and requirements of object must be implemented into measures. It means that integration safety and security measures could be in other category of object different and in the same category can be solving different too. This is reason for developing this proposal of system. Studies of dependencies are not possible, because this solving and decision making depends on expert's experiences and on specific conditions in object too. On the figure 4 is presented the proposal of system integration.

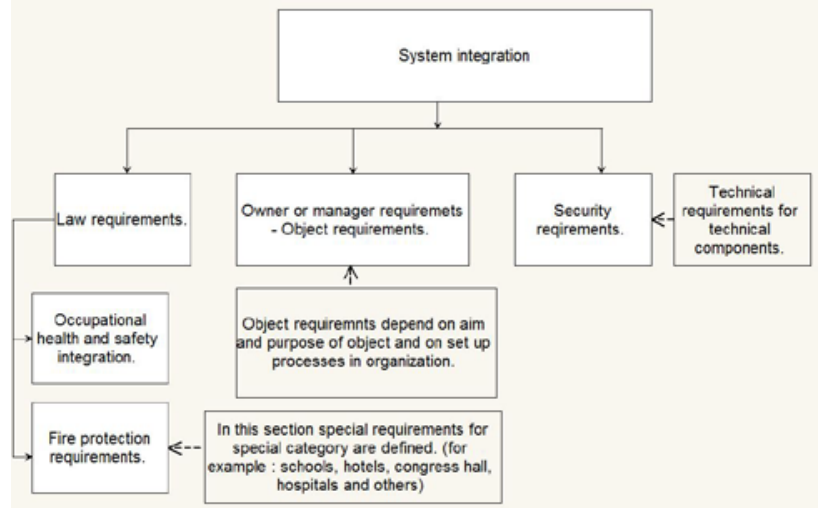

Figure 4. The proposal of system integration.

For proposal of solving incidents or security and safety situation we need to know about object next definition and characteristics:

- How is aim of object and how were processes set up?

- How is access managing? How is object divided?

- How are technical security components in object?

- Information about city and localization - it is called as External inputs parameters.

- Information about last incidents and problems.

- Information about human resources and job descriptions.

- $\quad$ And others.

After input parameters system can define requirements for object. The first and primary are law requirements. The next are security requirements, which are defined from level of security requirements. Level of security requirements experts can define after categorization object. Every category could have special parameters which are talked about situation and requirements and every object has one specific security number which is talked about situation in object only. The real situation is presented on figure 5 .

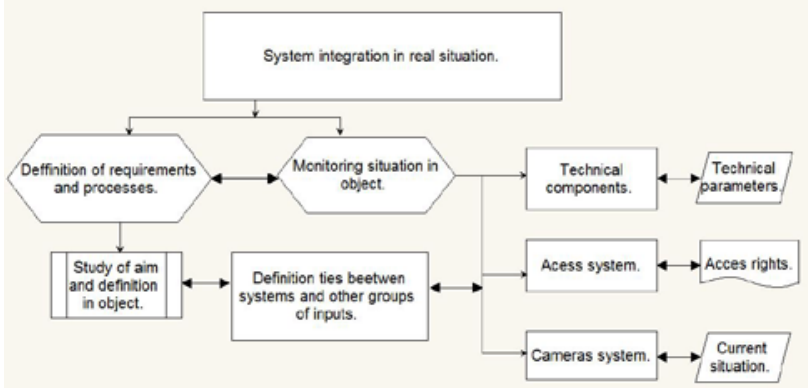

Figure 5. System integration process in real situation.

System integration in real situation is based on system solving and integration technical components to one system. This could achieved more effectivity of security and safety measurements.

If in the system will be implemented experiences from experts, system could be more intelligent and security manager needn't know more knowing in this system, but could be aimed to object requirements.

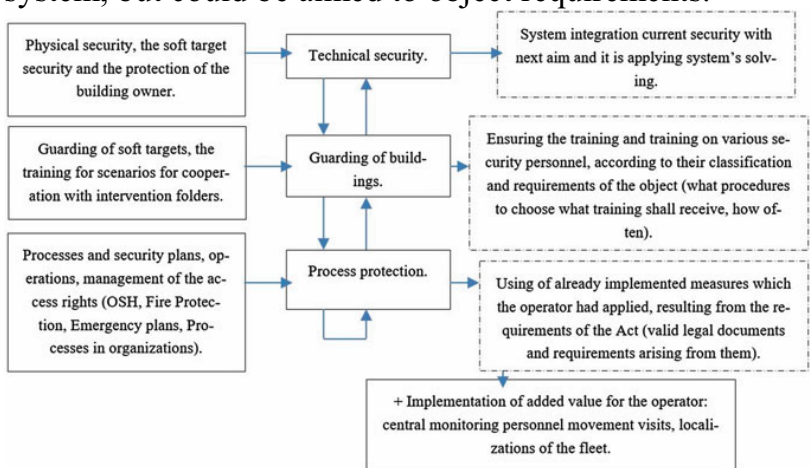

Figure 6. Implementation of experts experiencies. [2]

It is necessary to examine every object, but the aim is the examining and determining the security parameter. On the figure 6 is presented integration of technical security, guarding of building and process protection. The fuzzy logic can be used, because it necessary to know and work with a lot of plans and a lot of experts in one systems. It is a wide range of numerical values too, and a lot of technical components. It is important to define parameters and it is not about two values, but is about a lot of values, and it is reason for fuzzy logic use. This system will be adaptable with other methods, therefore every inputs can be set up and mechanism can be set up to. Then is the implementation of results from other group of security, for support solutions. [2]

\section{The other approach to solving}

Current situation is based on legislative requirements, which owners and managers must fulfilment. In the other hand next requirements are from owners and managers. It means requirements for achieving safety situation in object and to achieve avoiding to critical situations. Risk we could transport to other organization, accept or minimize to acceptable level. This system want to achieve maximize effect of measurements and achieve more effectivity of processes and no only security and safety processes. 
Developing special methods for system solution is one of next and supported action in process of developing this system. Special and next methods and approach have not effect if this methods are not supported by system integration. In this proposal will be implemented more methods and approaches which are specific for specific objects. The reason is simple. Every object has special conditions and special own requirements which result of aim of object. System should not define requirements which could negatively impact operation in object. [2]

In object are used software tools, which is specified for one group of safety and security requirement. For example for Fire protection it is software for authorized persons, which could help them with definition fire safety solving of building. Other systems are for security analysis and other for choosing correct technical components, for example. This proposed system is different, because it could help owners and managers more effectively choosing security and safety solution. System transformed expert's experiences to every object which have this software in management. System support making decision in every installed object. Eventual effect is effective solution and less financially depending. Requirements for operators are lower and it could eliminate problems which was identified in incidents. It was low level of security education and persons weren't trained for specified incidents. For owners and managers it could be tool for setting correct system in every law requirements and also in other requirements.

\section{Conclusion}

The paper describes proposed system for solving security and safety incidents in objects, which are categorized as soft targets. The system is based on software support and on expert's experiences. It is reason why was chosen fuzzy logic as operate language. Incidents in soft targets are different and solving this incidents require more groups of knowledges and experiences. This system could use more expert's experiences, and system doesn't have to solve mathematical dependencies of this statements. At the end paper is described current situation in soft targets and benefits which this solving could supply managers and owners by system.

\section{Acknowledgments}

This work was supported by the European Regional Development Fund under the project CEBIA-Tech Instrumentation No. CZ.1.05/2.1.00/19.0376 and also by the Internal Grant Agency of Tomas Bata University under the project No. IGA/FAI (CEBIA)/2016/12.

This work was supported by the research project VI20152019049 "RESILIENCE 2015: Dynamic Resilience Evaluation of Interrelated Critical Infrastructure Subsystems", supported by the Ministry of the Interior of the Czech Republic in the years 2015-2019 too.

\section{References}

1. F. Dernoncourt, Introduction to Fuzzy Logic (2013)

2. L. Duricova, M. Hromada, The Proposal of the Soft Targets Security.Advences in Intelligent Systems and Computing, Automation Control Theory Perspectives in Intelligent Systems, 3, 337-345 (2016)

3. G. J. Klir, B. Yuan, Theory and Application, 574 (1995)

4. L. Duricova, M. Hromada, Proceeding of 3rd CER Comparative, 2, 30-33 (2015)

5. F. Rosenberg, Nice Solutions for Critical Facilities (2014)

6. T. Takagi, M. Sugeno, Syst. Man. Cybern. 1, 116132 (1985)

7. J. Valouch, Int. J. Circ. Syst. Signal Process., 9, 205-2010 (2015)

8. L. A. Zadeh, Inf. Control, 8, 338-353 (1965) 